\title{
Consequences of non-uniform active currents in dendrites
}

\section{Terrence J. Sejnowski ${ }^{1,2 *}$}

1 Howard Hughes Medical Institute, Computational Neurobiology Laboratory, Salk Institute, La Jolla, CA, USA

2 Division of Biological Sciences, University of California San Diego, La Jolla, CA, USA

*Correspondence: terry@salk.edu

\section{A commentary on}

Modeling thalamocortical cell: impact of $\mathrm{Ca}^{2+}$ channel distribution and cell geometry on firing pattern

by Reza Zomorrodi, Helmut Kröger and Igor Timofeev

Thalamocortical (TC) neurons lead double lives: In a depolarized state near threshold excitatory synaptic input evokes regular spiking that faithfully "relays" information to the cortex, but when the cell is hyperpolarized, the same excitatory input can lead to a low-threshold spike (LTS), triggered by low the threshold calcium T-currents (Figure 1). LTSs in TC cells occur during sleep, when the membrane potential is hyperpolarized as a consequence of reduced levels of neuromodulatory inputs and the opening of potassium currents. The LTSs are prominent features of sleep spindles, but can also appear in states of alertness. We have known that TC cell have this dual function since it was first reported by Jahnsen and Llinás (1984), but only recently has the distribution of T-currents in the TC cells been explored.

T-current recorded from the somas of dissociated TC cells lacking dendrites have much smaller amplitudes than in cells

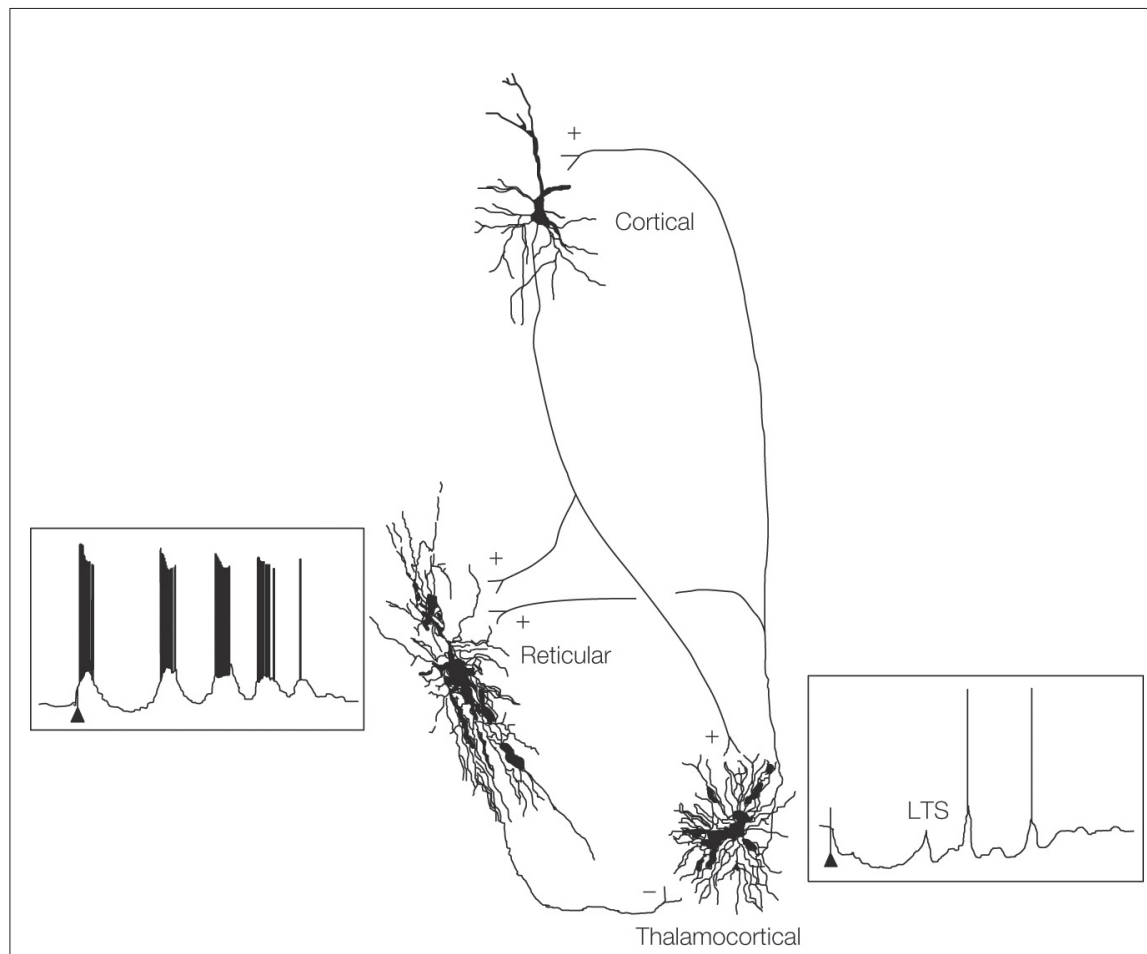

FIGURE 1 | Sleep, epilepsy and thalamic reticular inhibitory neurons. Steriade M. Cortical, reticular and thalamocortical neurons showing connectivity (+ excitation, - inhibition), intracellular recordings and anatomical reconstructions from cats. Cortical stimulation (arrows) leads to a sequence of spindle waves in the reticular cell and to a series of low-threshold spikes (LTS) in the thalamocortical cell. From Steriade (2005). recorded from thalamic slices, which suggests that the T-channels are mainly dendritic (Destexhe et al., 1998). Are the T-currents distributed uniformly in the dendrites, or are they concentrated more proximally or more distally? In this issue, Zomorrodi et al. (2008) have modeled these possibilities and concluded that the lowest threshold LTS occurs when the T-current are located proximally. Most of their simulations were based on a 3 compartment model, consisting of a soma, a proximal dendritic segment and a distal dendritic segment. They also reconstructed a complete TC cell and used simulations of the more accurate morphology to show that the results were even more robust than for the 3 compartment model. Confirmation of the prediction that $\mathrm{T}$-current is concentrated in the proximal dendrites of TC cells awaits high-resolution electron microscopic imaging of the $\mathrm{Ca}_{\mathrm{v}} 3.1$ and $\mathrm{Ca}_{\mathrm{v}} 3.3 \mathrm{~T}$-channels. In neurons of the reticular nucleus of the thalamus, which also exhibit an LTS, the $\mathrm{T}$-current is concentrated in the distal dendrites (Kovács et al., 2009), where they generate a prolonged LTS (Destexhe et al. 1996).

Although the proximal localization of T-currents in the dendrites of TC cells may require the least current to trigger an LTS when current is injected in the soma, it is not clear whether this is also true for synaptic conductance changes in the dendrites. Another open issue is whether single dendrites behave like functional units, as occurs in hippocampal cells (Gasparini et al., 2004)? If so, input from a cluster of synapses on a single dendrite could be sufficient to trigger an LTS. Or is the TC cell electrically compact? In a previous compartmental model of the TC cell (Destexhe et al., 1998), reconstructed from a $200 \mu \mathrm{m}$ slice, the dendrites were much shorter and more electrically compact than the reconstructed TC cell in the current study, which had dendrites extending $400 \mu \mathrm{m}$ from the soma (Zomorrodi et al., 2008).

The H-current, a non-specific cation current that is activated by membrane hyper- 
polarization, also participates in the LTS in TC cells, particularly during sleep spindles. The effects of its distribution within the TC cell could also be studied with compartmental models, in conjunction with the distribution of the T-currents. The optimal distributions of these currents may not be independent of each other, so they need to be jointly varied.

The calcium that enters the neuron during an LTS must be internally bound or extruded to maintain the equilibrium of free calcium in the cell in the long term. This is accomplished by $\mathrm{Ca}^{2+} / \mathrm{Na}^{+}$ metabolic exchangers in the plasma membrane. Reducing the number of T-channels needed to trigger an LTS would reduce the number of calcium ions that need to be extruded later, and hence would reduce the energy that the TC cell must expend to function. In the hippocampus, nonmyelinated axons have a fast sodium current and delayed potassium current, which reduces the overlap of the currents and minimizes the cost of an action potential (Alle et al., 2009). Similarly, pyramidal neurons and fast-spiking interneurons in the cerebral cortex also minimize energy expenditure for the patterns of action potentials they generate in vivo (Hasenstaub et al. 2009). This may be a general principle for neural information processing systems (Laughlin et al., 1998; Laughlin and Sejnowski, 2003).

\section{REFERENCES}

Alle, H., Roth, A., and Geiger, J. R. P. (2009). Energyefficient action potentials in hippocampal mossy fibers. Science 325, 1405-1408.

Destexhe, A., Contreras, D., Steriade, M., Sejnowski, T. J., Huguenard, J. R. (1996). In vivo, In vitro, and computational analysis of dendritic calcium currents in thalamic reticular neurons, J. Neurosci. 16, 169-185.

Destexhe, A., Neubig, M., Ulrich, D., and Huguenard, J. (1998). Dendritic low threshold calcium currents in thalamic relay cells. J. Neurosci. 18, 3574-3588.

Gasparini, S., Migliore, M., and Magee, J. C. (2004). On the initiation and propagation of dendritic spikes in CA1 pyramidal neurons. J. Neurosci. 24, 11046-11056.

Hasenstaub, A., Otte, S., Callaway, E., and Sejnowski, T. J. (2009). Metabolic constraints on the biophysics of action potential generation, Neuron.

Jahnsen, H., and Llinás, R. (1984). Electrophysiological properties of guinea-pig thalamic neurones: an in vitro study. J. Physiol. 349, 205-226.
Kovács, K., Sik, A., Ricketts, C., and Timofeev, I. (2009). Subcellular distribution of low-voltage activated T-type $\mathrm{Ca}(2+)$ channel subunits $(\mathrm{Ca}(\mathrm{v}) 3.1$ and $\mathrm{Ca}(\mathrm{v}) 3.3)$ in reticular thalamic neurons of the cat. J. Neurosci. Res. [Epub ahead of print].

Laughlin, S. B., and Sejnowski, T. J. (2003). Communication in Neuronal Networks, Science 301, 1870-1874.

Laughlin, S. B., de Ruyter van Steveninck, R. R., and Anderson, J. C. (1998). The metabolic cost of neural information. Nat. Neurosci. 1, 36-41.

Steriade, M. (2005). Sleep, epilepsy and thalamic reticular inhibitory neurons. Trends Neurosci. 28, 317-324.

Zomorrodi, R., Kröger, H., and Timofeev, I. (2008). Modeling thalamocortical cell: impact of $\mathrm{Ca} 2+$ channel distribution and cell geometry on firing pattern. Front. Comput. Neurosci. 2, 1-11. doi: 10.3389/ neuro.10.005.2008.

Received: 10 November 2009; published: 15 December 2009

Citation: Front. Neurosci. (2009) 3, 3: 332-333. doi: 10.3389/neuro.01.038.2009

Copyright (c) 2009 Sejnowski. This is an open-access publication subject to an exclusive license agreement between the authors and the Frontiers Research Foundation, which permits unrestricted use, distribution, and reproduction in any medium, provided the original authors and source are credited.

\section{A new phase for two-photon microscopy}

\section{Timothy E. Holy* \\ Department of Anatomy and Neurobiology, Washington University School of Medicine, St Louis, MO, USA \\ *Correspondence: holy@wustl.edu}

\section{A commentary on}

SLM microscopy: scanless two-photon imaging and photostimulation using spatial light modulators

by Volodymyr Nikolenko, Alan Woodruff, Brendon Watson, Rafael Yuste, Darcy Peterka and Roberto Araya

Two-photon excitation has enabled remarkable advances in our understanding of the nervous system. Its three-dimensional resolution, superior depth penetration, and minimal phototoxicity for out-of-focus portions of the sample have made it the technique of choice for an impressive array of applications. For all of its advantages, however, it is not without its limitations; for neuroscience, perhaps chief among these is the time it takes to collect a large image over a field of view containing many neurons. To address this limitation, Nikolenko et al. (2008) have provided a new method for creating customized two-photon excitation, thereby allowing the user to conduct photostimulation or imaging from tens of neurons simultaneously.

Conventional two-photon microscopy assembles an image by scanning a single laser spot over the sample. While there are fast-scanning microscopes that can collect an entire frame in 1/30 of a second, these instruments are hampered by high noise levels. The dominant source of noise is not the instrument itself: instead, it arises from the limited number of photons that are collected in each pixel. Reducing the noise simply requires more photons-but under typical conditions, each illuminated fluorophore emits photons at its maximal rate, which means that the only way to get more photons is to integrate over longer times.

Efforts to circumvent this limit have mostly taken one of two approaches (see Conchello and Lichtman, 2005; Wilt et al., 2009 for reviews). Some instruments illuminate many pixels simultaneously, satisfying the integration time for many pixels in parallel. The others carefully choose a restricted set of "interesting" pixels, thereby reducing the total number of pixels that need to be acquired during each scan. 